\title{
Análise das propriedades ópticas da água do reservatório Rodolfo Costa e Silva - Itaara, RS, Brasil, usando dados espectrais de campo e imagens orbitais multiespectrais
} (doi:10.4136/ambi-agua.28)

\author{
Fábio Marcelo Breunig ; Flávio Wachholz²; Waterloo Pereira Filho ; Conrado de \\ Moraes Rudorff ${ }^{4}$ \\ Instituto Nacional de Pesquisas Espaciais - Mestrando em Sensoriamento Remoto \\ E-mail: fabiobreunig@gmail.com \\ ${ }^{2}$ Universidade Federal de Santa Maria - Mestrando em Geografia \\ E-mail: fwalemao@gmail.com \\ ${ }^{3}$ Universidade Federal de Santa Maria - Docente do Departamento de Geociências \\ E-mail: waterloopf@gmail.com \\ ${ }^{4}$ Instituto Nacional de Pesquisas Espaciais - Mestre em Sensoriamento Remoto \\ E-mail: cmr@ltid.inpe.br.
}

\section{RESUMO}

O trabalho avaliou a separabilidade de classes de água, a partir de técnicas de análises da reflectância de superfície, com dados medidos em campo e imagens multiespectrais adquiridas em nível orbital. A área de estudo foi o reservatório Rodolfo Costa e Silva, localizado na região central do Rio Grande do Sul. A metodologia foi baseada na coleta de dados in situ - total de sólidos em suspensão, clorofila $(a, b, c)$, transparência da água e espectros de reflectância bidirecional - em 21 pontos amostrais no dia 16 de maio de 2006 . A técnica de remoção do contínuo foi aplicada aos espectros de campo em quatro intervalos de bandas de absorção: $400-550 \mathrm{~nm}, 610-640 \mathrm{~nm}, 650-680 \mathrm{~nm}$ e $580-700 \mathrm{~nm}$. Os parâmetros de remoção do contínuo das bandas de absorção analisados foram: profundidade, área e largura. Verificou-se que os parâmetros de profundidade, área e a largura da banda de absorção não apresentaram potencial de separabilidade em classes espectrais relacionadas à pequena variação na concentração dos componentes opticamente ativos na água. Imagens CBERS2/CCD e Landsat 5/TM adquiridas em datas próximas à atividade de campo foram corrigidas dos efeitos atmosféricos e classificadas. Os resultados das classificações não apresentaram correlações significativas com os parâmetros de qualidade da água, a fim de proporcionar uma caracterização espectral das classes de água ou compartimentos. A dificuldade no estabelecimento de relações entre os parâmetros de reflectância espectral e qualidade da água foi devida, principalmente, à baixa variabilidade dos componentes opticamente ativos na água do Reservatório Rodolfo Costa e Silva. Neste caso, as análises espectrais testadas neste trabalho não foram sensíveis às pequenas variações observadas por meio dos dados de campo.

Palavras-chave: Sensoriamento remoto; reservatórios; remoção do contínuo.

\section{Analysis of optical proprieties of the water reservoir Rodolfo Costa e Silva - Itaara, RS, Brazil, with field spectral data and orbital multispectral images}

\section{ABSTRACT}

An evaluation of the discrimination of water classes using continuum removal technique applied over spectral data obtained in field and multispectral images classification is 
presented. The study area was the Rodolfo Costa e Silva water reservoir, located in central region of Rio Grande do Sul (RS) State, in Southern region of Brazil. The methodology was based on in situ data collection of: total suspended solids, chlorophyll (a, b and c), water transparency, and bidirectional spectral reflectance. These data were collected in 21 point (samples) in May 16, 2006. The continuum removal technique was applied on the spectral data over 4 absorption bands: $400-550 \mathrm{~nm}, 610-640 \mathrm{~nm}, 650-680 \mathrm{~nm}$ e $580-700 \mathrm{~nm}$. The continuum removal parameters analyzed for each absorption band were: depth, area and width. The multispectral images used were CBERS-2/CCD and Landsat 5/TM. The images were acquired in a date nearest to field work and with appropriate weather conditions. These images were corrected by removing atmospheric effects and then classified. According to the results obtained from the continuum removal technique, it was verified that band depth, area and width did not present a good potential to separate different water classes. Digital classification results did not show significant correlations with the limnological parameters collected in field and, therefore, could not be used to characterize spectrally different water classes or compartments. The main problem of establishing relationships between spectral reflectance and water quality parameters was due to the low variability of optical components in the water of Rodolfo Costa e Silva Reservoir. In this case the spectral analyses (considering both techniques) were not sensitive to the relative small variations observed in field data.

Keywords: Remote sensing; reservoirs; continuum removal.

\section{INTRODUÇÃO}

O reservatório Rodolfo Costa e Silva, atualmente vinculado a Companhia Riograndense de Saneamento (CORSAN), é uma das fontes de água usadas para abastecer a cidade de Santa Maria, no centro do Rio Grande do Sul. A qualidade da água desse reservatório tem implicações diretas no processo de tratamento e no custo da água. Vários fatores influenciam na qualidade da água das barragens, dentre os quais se destacam os processos agrícolas, o desmatamento, urbanização, indústrias, mineração e o sistema de transportes (Jobin, 1998). Os impactos sobre os lagos, reservatórios e rios são inúmeros, destacando-se a variação da temperatura, oxigênio dissolvido, total de sólidos em suspensão, concentração de nutrientes, modificação da hidráulica, aumento da concentração de mercúrio e ação dos gases do efeito estufa (NRC, 1993, 2002; Norling et al., 2004).

O estudo de reservatório a partir da espectroradiometria e de imagens de satélite constitui uma nova forma de acessar as características limnológicas. Ao considerar a extensão e quantidade de corpos hídricos no Brasil, fica evidente a necessidade de novas ferramentas para monitorar a dinâmica e características desses ambientes. A isso se soma o fato de as barragens terem relevância quanto à produção de energia no Brasil (Jobin, 1998). Devemos atentar ainda para o fato da construção de numerosas barragens e açudes, para servirem de reservatório de água (Esteves, 1998).

A presença de sedimentos nos lagos é importante para a avaliação do ecossistema, estudo da evolução e interações que ocorrem entre o sistema terrestre e aquático, e para avaliar os impactos ambientais. Os sedimentos límnicos podem ser orgânicos ou inorgânicos. Os sedimentos orgânicos em suspensão são representados pelo fitoplâncton, detritos e microorganismos vivos. Esses compostos possuem menor condutividade e baixa resistência dielétrica. Os sedimentos inorgânicos em suspensão são representados por partículas trazidas das áreas de captação do reservatório ou drenagem, representados por minerais (Esteves, 1998). 
BREUNIG, F. M.; WACHHOLZ F.; PEREIRA FILHO, W.; RUDORFF, C. M. Análise das propriedades ópticas da água do reservatório Rodolfo Costa e Silva - Itaara, RS, Brasil, usando dados espectrais de campo e imagens orbitais multiespectrais. Ambi-Agua, Taubaté, v. 2, n. 2, p. 88-102, 2007. (doi:10.4136/ambi-agua.28)

O uso do sensoriamento remoto nos estudos limnológicos possibilita avaliar grandes áreas e relacionar a resposta espectral da água com dados limnológicos obtidos em campo (Dekker, 1993). A eficiência dessa ferramenta foi comprovada em vários estudos, pela utilização de imagens multiespectrais (Novo et al., 2006) e hiperespectrais (Galvão et al., 2003; Rudorff et al., 2006), além de dados espectrais obtidos em campo e laboratório.

O sensoriamento remoto permite estimar a transparência da água, a concentração de sedimentos em suspensão e a quantidade de clorofila, identificar o estado trófico do meio, quantificar e mapear plantas submersas e emersas (Pasterkamp et al., 1999; Novo, 2001). Entretanto, é preciso considerar que o comportamento espectral da água em geral é determinado pela absorção das algas, o espalhamento provocado por moléculas de água nas ondas curtas e o espalhamento de Raman nas ondas intermediárias, além da fluorescência. Nos estudos de ambientes aquáticos continentais essas características somam-se ao retroespalhamento, a absorção em ondas curtas do visível, e ao decréscimo do espalhamento molecular, Raman e da contribuição da fluorescência. Isso torna o estudo de corpos hídricos terrestres mais complexos e cautelosos (Dekker, 1993).

Em um estudo comparando os resultados estimados com uso de sensoriamento remoto e resultados de algoritmos, Melack e Gastil (1994) verificaram que para estimar a quantidade de clorofila é necessária a confirmação por meio de sensores remotos de alta resolução espectral. Por outro lado, Dekker et al. (1991) estimaram a qualidade da água de ambientes aquáticos continentais pelo sistema MSS, sugerindo o uso das faixas entre 600 e $720 \mathrm{~nm}$ para estimar a quantidade de detritos orgânicos, algas e matéria dissolvida.

Numa breve abordagem sobre a aplicação do sensoriamento remoto à hidrografia, Garcia (1982) comenta que a quantidade de sedimentos carreados para as represas de hidroelétricas é um fenômeno que pode ser estudado pelo uso de sensoriamento remoto. Dekker et al. (2001) comentam que medidas de matéria em suspensão incluem o peso seco do séston, inclusive a matéria inorgânica, como silte e argila, e matéria orgânica incluindo detritos e algas. A quantidade de sólidos em suspensão determina uma variação positiva na reflexão em todas das faixas, isso está relacionado ao aumento do retroespalhamento.

O aumento da concentração de sólidos em suspensão ocasiona um aumento da reflectância em direção aos comprimentos de onda mais longos. Entre a faixa dos 500 e 700 nm, a reflectância se mantém praticamente constante, apesar de mais elevada. Ainda é verificado um aumento em direção ao infravermelho (Novo, 2001). De acordo com Pereira Filho (1991), a própria granulometria dos sólidos em suspensão pode afetar a reflexão do corpo de água.

Existe uma relação entre a profundidade do disco de Secchi e a concentração de matéria dissolvida e materiais sólidos em suspensão, que pode ser correlacionada com dados de sensoriamento remoto (Dekker, 1993). A profundidade do disco de Secchi depende do coeficiente de atenuação da água. Esse coeficiente pode ser escrito em função das propriedades inerentes da água (Pasterkamp et al., 1999).

A técnica de remoção do contínuo foi proposta por Clark e Rough (1984) e consiste em remover as feições contínuas dos espectros. O contínuo é representado por uma função matemática utilizada para isolar bandas de absorção particulares dos espectros de reflectância além de acentuar as feições (Mutanga; Skidmore, 2003). Em última instância, consiste numa normalização de dados espectrais que permite reduzir os efeitos externos (Van der Meer, 2000; Clark; Roush, 1984)

Neste contexto, o objetivo do trabalho foi avaliar o potencial de caracterização das propriedades ópticas da água a partir da técnica de remoção do contínuo aplicada sobre os dados espectrais coletados em campo e da classificação de imagens multiespectrais orbitais. 


\section{MATERIAL E MÉTODOS}

A área de captação do reservatório Rodolfo Costa e Silva é formada pela bacia hidrográfica do rio Ibicuí-Mirim. Está localizado no planalto do Sul-riograndense, onde as características da estrutura basáltica se refletem no tipo de solo avermelhado. A bacia de captação do reservatório possui 4.880 hectares, onde predominam vertentes suaves, representadas por colinas. O uso da terra predominante na bacia é agrícola, apresentando também áreas de campo destinadas à pecuária, e pequenas áreas de vegetação arbórea, especialmente de matas ciliares. A localização do reservatório pode ser vista na Figura 1.

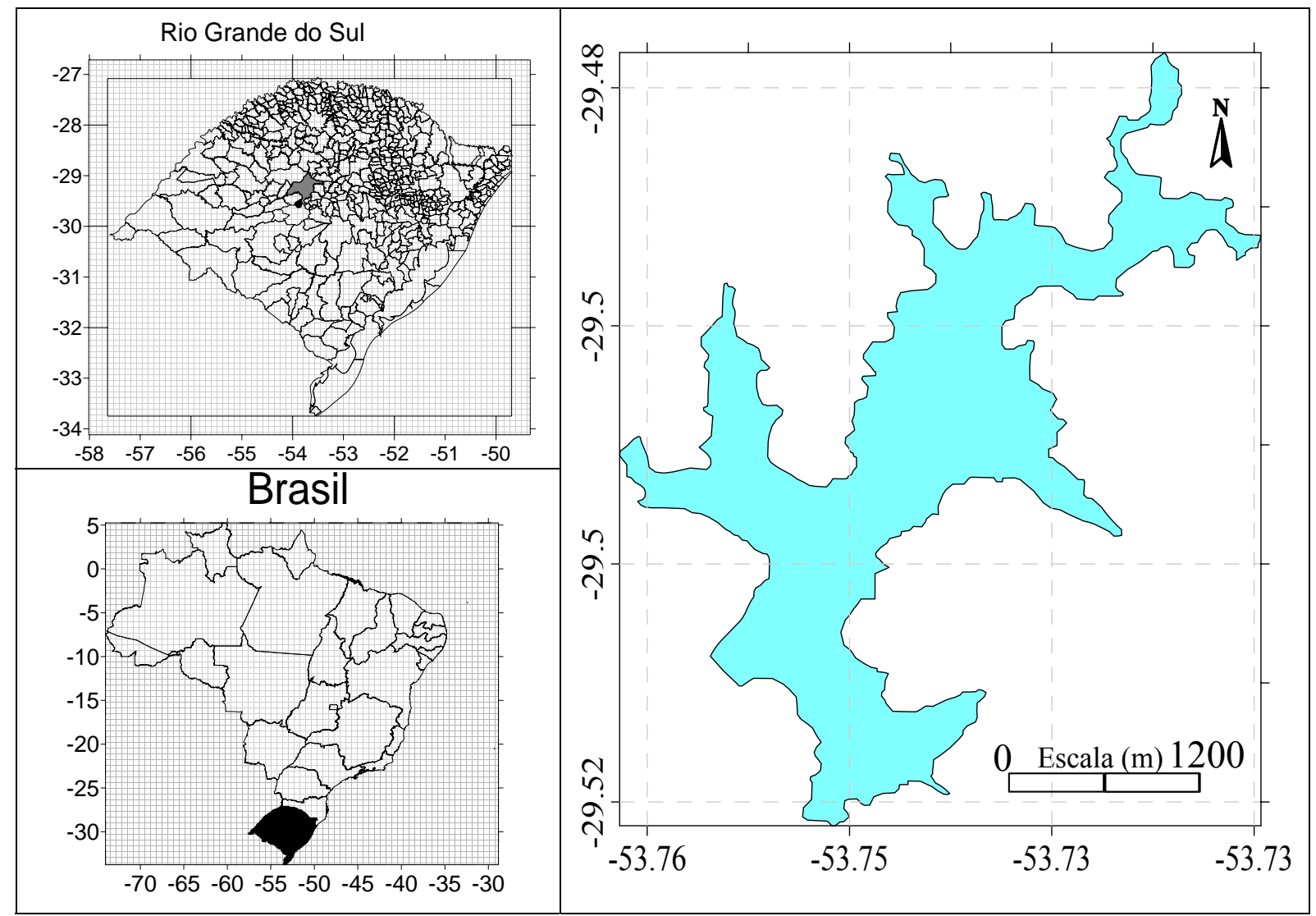

Figura 1. Localização do reservatório de água Rodolfo Costa e Silva.

Os dados de campo foram coletados no dia 16 de maio de 2006, em 21 pontos amostrais, distribuídos geograficamente no reservatório (Figura 2), de forma a cobrir o reservatório de forma relativamente homogênea.

Foram avaliados os seguintes parâmetros limnológicos: transparência da água por meio da profundidade do Disco de Secchi (SD); Total de Sólidos em Suspensão (TSS), segundo metodologia descrita por CETESB (São Paulo, 1987) e; clorofila a, b e c, de acordo com Nush (1980). Os parâmetros radiométricos avaliados foram: espectros de reflectância bidirecional obtidos pelo espectroradiômetro FieldSpec Hand Held no intervalo espectral de 400 a 900 nm e imagens de satélite do CBERS-2 (20-04-2006) e Landsat TM-5 (5-05-2006).

As condições de tempo para a coleta de dados foram adequadas, ou seja, com sol, ausência de vento ou com brisa e com a superfície da água calma ou com pequenas ondulações, sendo o vento um dos fatores mais importantes na degradação das informações 
espectrais em coletas de campo (Pereira Filho et al., 2005). Para a redução de ruídos nos dados espectrais coletados em campo, foi aplicado um filtro de média móvel de 11 pontos.

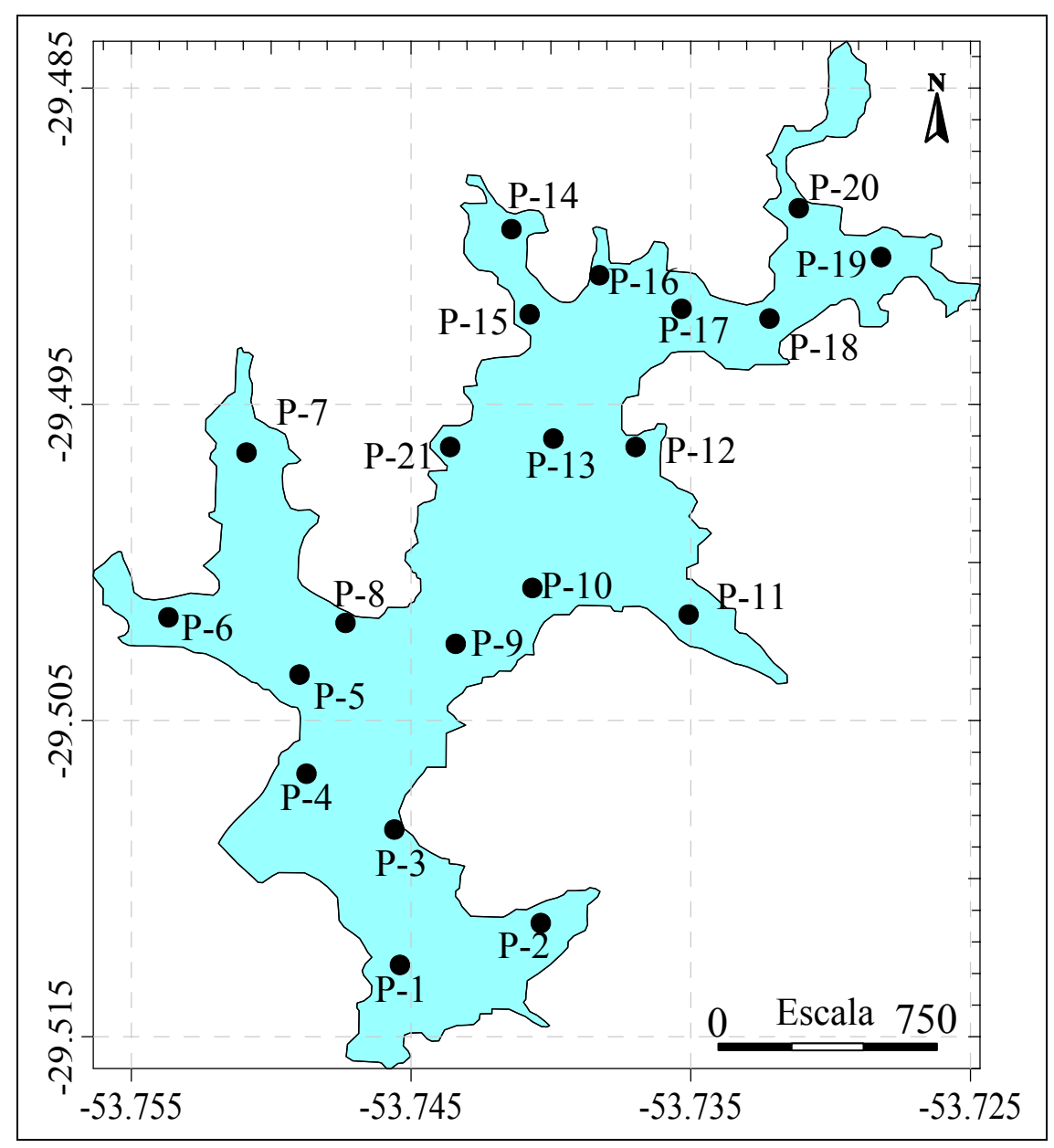

Figura 2. Localização dos 21 pontos amostrais no reservatório.

A correção atmosférica das imagens CBERS-2 CCD foi feita por meio do código $5 \mathrm{~S}$ (Vermote et al., 1997). As imagens Landsat 5 TM foram corrigidas pelo modelo MODTRAN implementado no Flaash-ENVI (ENVI, 2004). As imagens resultantes foram convertidas para reflectância de superfície. As imagens de reflectância foram classificadas no aplicativo ENVI pelo classificador IsoData (ENVI, 2004). O número de classes foi definido para permanecer entre 3 e 5 classes, considerando a mínima variância dos dados de campo previamente avaliada

A formulação matemática para a remoção do contínuo dos espectros de reflectância é apresentada na Equação 1 (Clark; Rough, 1984; Galvão, 2001; Hong et al., 2004). Cabe destacar que o espectro do contínuo pode ser formulado matematicamente pela equação da reta, definida pelos coeficientes angular e linear.

$\rho_{C R(\lambda)}=\frac{\rho_{\text {Original }(\lambda)}}{\rho_{\text {contínuo }(\lambda)}}$

Em que $\rho_{C R \lambda}$ é o fator de reflectância direcional cônico com o contínuo removido; $\rho_{\text {Original }(\lambda)}$ é o fator de reflectância direcional cônico original; $\rho_{\text {contínu }(\lambda)}$ é o espectro do contínuo e $\lambda$ 
representa o comprimento de onda. Para se obter a profundidade de banda $P_{d}$, utiliza-se a Equação 2 (Kokaly; Clark, 1999; Noomem et al., 2005).

$P_{d}=\left(1-\rho_{C R \lambda}\right)$

A escolha dos limites das bandas de absorção deve ser feita de modo a ressaltar as feições de absorção. Os limites das bandas de absorção e o comprimento de onda $(\lambda)$ central escolhidos como referência neste trabalho são mostrados na Tabela 1.

Tabela 1. Bandas de absorção e $\lambda$. central de cada banda.

\begin{tabular}{lcccc}
\multicolumn{1}{c}{ Banda de absorção } & $\begin{array}{c}\lambda \\
\text { Central }\end{array}$ & $\begin{array}{c}\lambda \\
\text { Limite inferior }\end{array}$ & $\begin{array}{c}\lambda \\
\text { Limite superior }\end{array}$ \\
\hline $1^{\mathrm{a}}$ banda de absorção & 450 & 400 & 550 \\
$2^{\mathrm{a}}$ & banda de absorção & 630 & 610 & 640 \\
$3^{\mathrm{a}}$ banda de absorção & 630 & 580 & 700 \\
$4^{\mathrm{a}}$ & banda de absorção & 675 & 650 & 680 \\
\hline
\end{tabular}

A área das bandas de absorção $\left(A_{P}\right)$ foi calculada pela aproximação numérica dada pela Equação 3. Por fim, a largura das bandas de absorção é dada pela determinação das posições anterior e posterior ao $\lambda$ de maior profundidade referentes à metade do máximo (Pu et al., 2003).

$A_{P}=\sum_{i=m}^{M} \frac{\left(\rho_{i}+\rho_{i-1}\right)}{2} \cdot\left(\lambda_{i}-\lambda_{i-1}\right)$

em que $i$ representa o intervalo de cálculo do limite inferior $m$ ao limite superior da banda $(M)$.

Em relação às análises estatísticas, estas foram baseadas no cálculo da média, desvio padrão, variância e correlação de Pearson (5 \% de significância).

\section{RESULTADOS E DISCUSSÃO}

A forma dos espectros da água fornece indicativos de quais componentes opticamente ativos estão dominando o espectro. A Figura 3 ilustra os espectros de reflectância bidirecional obtidos nos 21 pontos amostrais. Todos eles apresentam maior reflectância bidirecional na faixa espectral referente ao verde, em que a clorofila é o principal componente que influencia no processo de espalhamento (Kirk, 1996).

A baixa variabilidade dos dados e baixa porcentagem de reflectância restringem a análise direta com dados ópticos (reflectância) coletados em campo. Portanto, foi utilizada a técnica de remoção do contínuo para realçar as diferenças em quatro bandas de absorção, especialmente relacionadas à presença de clorofila na água, pois, normalmente, elas são proporcionais a sua concentração (Kirk, 1996).

Apesar dos dados terem sido filtrados, às vezes, os ruídos se sobressaem. Isso sugere que a variabilidade nos espectros dos diferentes pontos pode estar relacionada à precisão das medidas radiométricas e à influência atmosférica. Esse foi o resultado encontrado para a primeira banda de absorção analisada (400-550 nm).

A variabilidade da profundidade da banda de absorção entre 400 e $550 \mathrm{~nm}$ no comprimento de onda de $450 \mathrm{~nm}$ (tomado como referência para esta banda) foi baixa. A 
maior variação foi relacionada ao ponto P-20. Analisando os valores do desvio padrão e da variância, respectivamente de 0,02971 e 0,0004, verifica-se que a separação de diferentes tipos de classes de água a partir de imagens de satélite é bastante prejudicada, visto que as variações não são significativas.

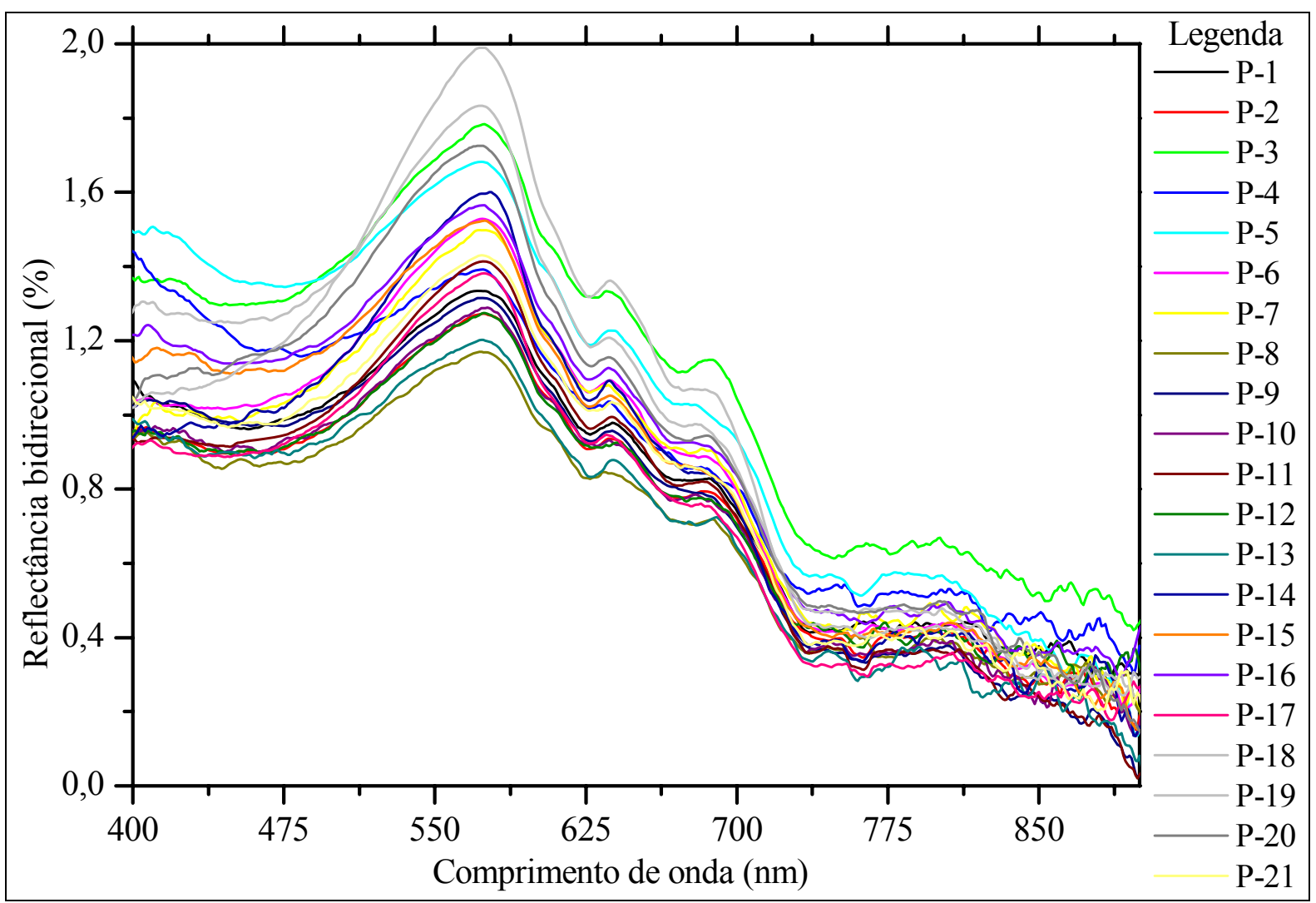

Figura 3. Espectros de reflectância bidirecional coletados em campo.

Considerando variabilidade da área da banda de absorção entre 400 e $550 \mathrm{~nm}$, constatouse que o ponto P-6 apresenta maior variação em relação à média da área. Novamente a variância e o desvio padrão foram baixos. Entretanto, deve-se considerar que as imagens analisadas neste trabalho não são da mesma data da obtenção dos espectros de reflectância bidirecional, contudo, obtiveram-se as imagens mais próximas possível, considerando a influência de nuvens e a data de passagem dos satélites CBERS-2 e Landsat-5.

A análise da segunda banda de absorção $(610-640 \mathrm{~nm})$ mostra que a profundidade de banda não permitiu identificar variações significativas nos espectros (Figura 4). Nessa banda, os pontos amostrais P-3, 4 e 7 apresentaram as menores absorções relacionadas à menor concentração de Clorofila c (Figura 7). A maior profundidade nos pontos P-13 e P-9 indica uma maior presença de clorofila, concordando com os resultados apresentados na Figura 7.

A Figura 5A mostra a variação da profundidade das bandas de absorção em $630 \mathrm{~nm}$ (para o intervalo de 610-640 nm). Verifica-se que a variabilidade dos dados é baixa, novamente indicando dificuldade na classificação das imagens de satélite, em especial a banda do vermelho. A Figura 5B, refere-se à subtração da profundidade de cada ponto da profundidade média do conjunto.

O desvio em relação à média dos parâmetros profundidade e área da banda de absorção entre 610-640 nm mostra que os pontos P-3, 4 e 7 se destacam com menores índices de absorção de radiação. Esse resultado mostra que tanto a área quanto a profundidade de banda produzem resultados similares e explica a alta correlação (97\%) entre esses dois parâmetros. 
Cabe destacar que isso vale para esta banda de absorção. O desvio padrão e a variância para a área de profundidade de banda não foram significativos.

A terceira banda de absorção $(650-680 \mathrm{~nm})$ compreende a faixa que sofre forte influência do TSS e, portanto, poderia ser utilizada para estimar variações desse componente opticamente ativo. O comprimento de onda central adotado para esse intervalo foi $675 \mathrm{~nm}$, e o resultado da profundidade de banda mostrou que a menor absorção aconteceu nos pontos amostrais P-4, 7 e 9, indicando maior concentração de TSS.

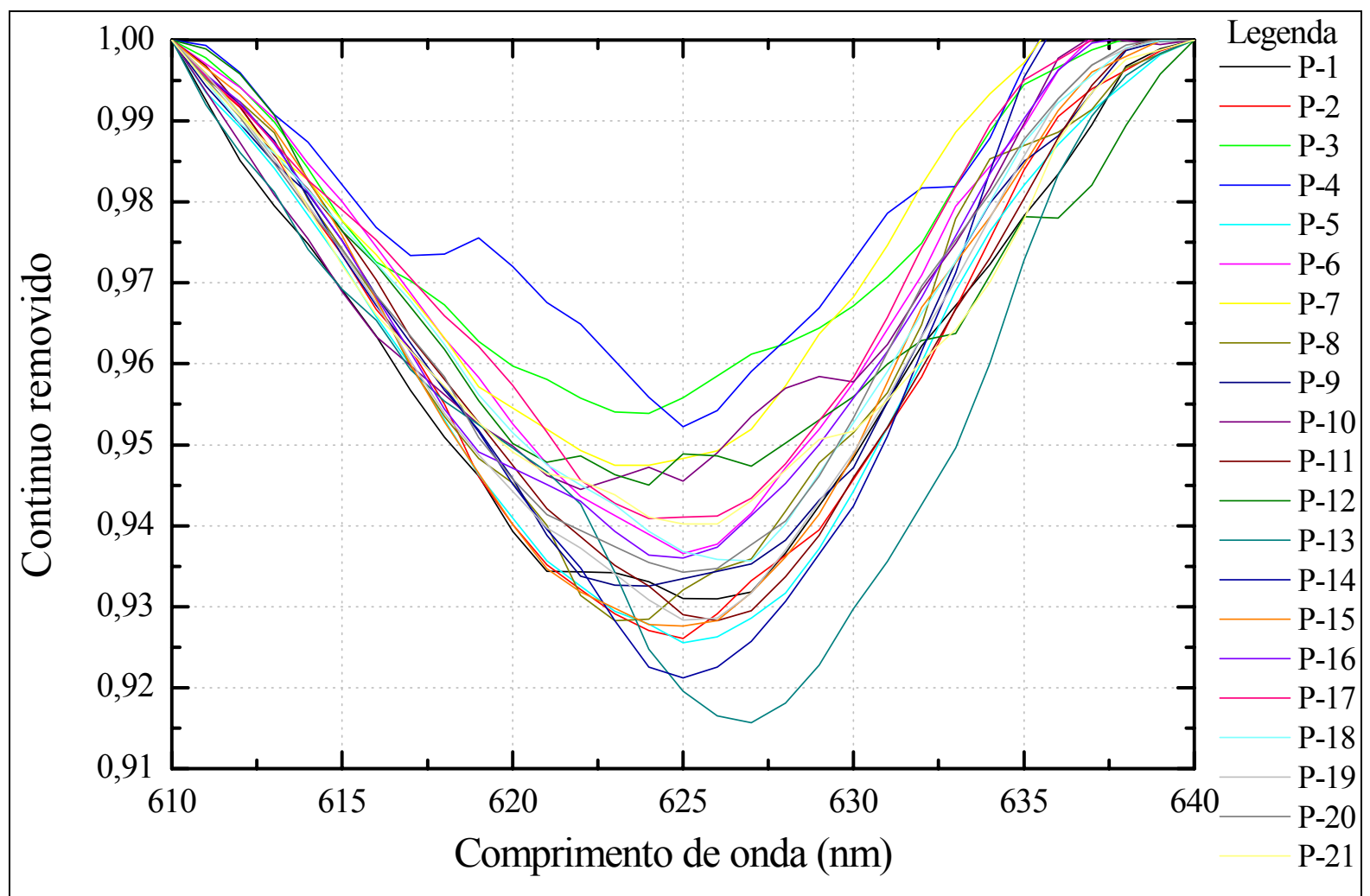

Figura 4. Remoção do contínuo aplicado à faixa espectral de 610-640 nm.

Por outro lado, os pontos P-10, 11 e 19 mostraram maior profundidade e, conseqüentemente, uma maior penetração da radiação na água e menor reflectância bidirecional. Entretanto, ao correlacionar os dados de profundidade de banda com o TSS (valores de TSS e SD são apresentados na Tabela 2), os resultados indicaram baixa correlação, fato que pode estar associado à baixa variabilidade dos dados, o que os torna mais susceptíveis aos erros de medida. O desvio padrão da profundidade da banda de absorção centrada em $675 \mathrm{~nm}$ foi de 0,0068 e sua variância foi de 0,000046, comprovando sua baixa variabilidade. Considerando o parâmetro área, as estatísticas também indicaram pouca variação.

Avaliando a correlação entre os dados da profundidade de banda e área da banda de absorção, verificou-se um coeficiente de $64 \%$, o que indica que os dois parâmetros podem produzir resultados distintos nessa banda e requerem cuidado ao serem utilizados, a fim de evitar erros. Esse fato recai especialmente quando estão sendo estudados corpos hídricos de baixa variabilidade dos componentes opticamente ativas.

Para a última banda de absorção estudada $(580-700 \mathrm{~nm})$, os resultados não indicaram grandes variações da reflectância. A análise da profundidade de banda de absorção entre 580 $\mathrm{nm}$ e $700 \mathrm{~nm}$, permitiu verificar que não existem variações significativas nos pontos 
BREUNIG, F. M.; WACHHOLZ F.; PEREIRA FILHO, W.; RUDORFF, C. M. Análise das propriedades ópticas da água do reservatório Rodolfo Costa e Silva - Itaara, RS, Brasil, usando dados espectrais de campo e imagens orbitais multiespectrais. Ambi-Agua, Taubaté, v. 2, n. 2, p. 88-102, 2007. (doi:10.4136/ambi-agua.28)

amostrados. Cabe destacar que os pontos P-17 e P-18, apresentaram as maiores variações. O desvio padrão calculado sobre esse parâmetro foi de 0,016704 , e a variância de 0,000279 . Esse baixo valor de variância indica a pequena variabilidade dos dados. Ainda considerando a quarta banda de absorção, a área também não apresentou resultados que incrementassem a separabilidade de classes de água no reservatório Rodolfo Costa e Silva.
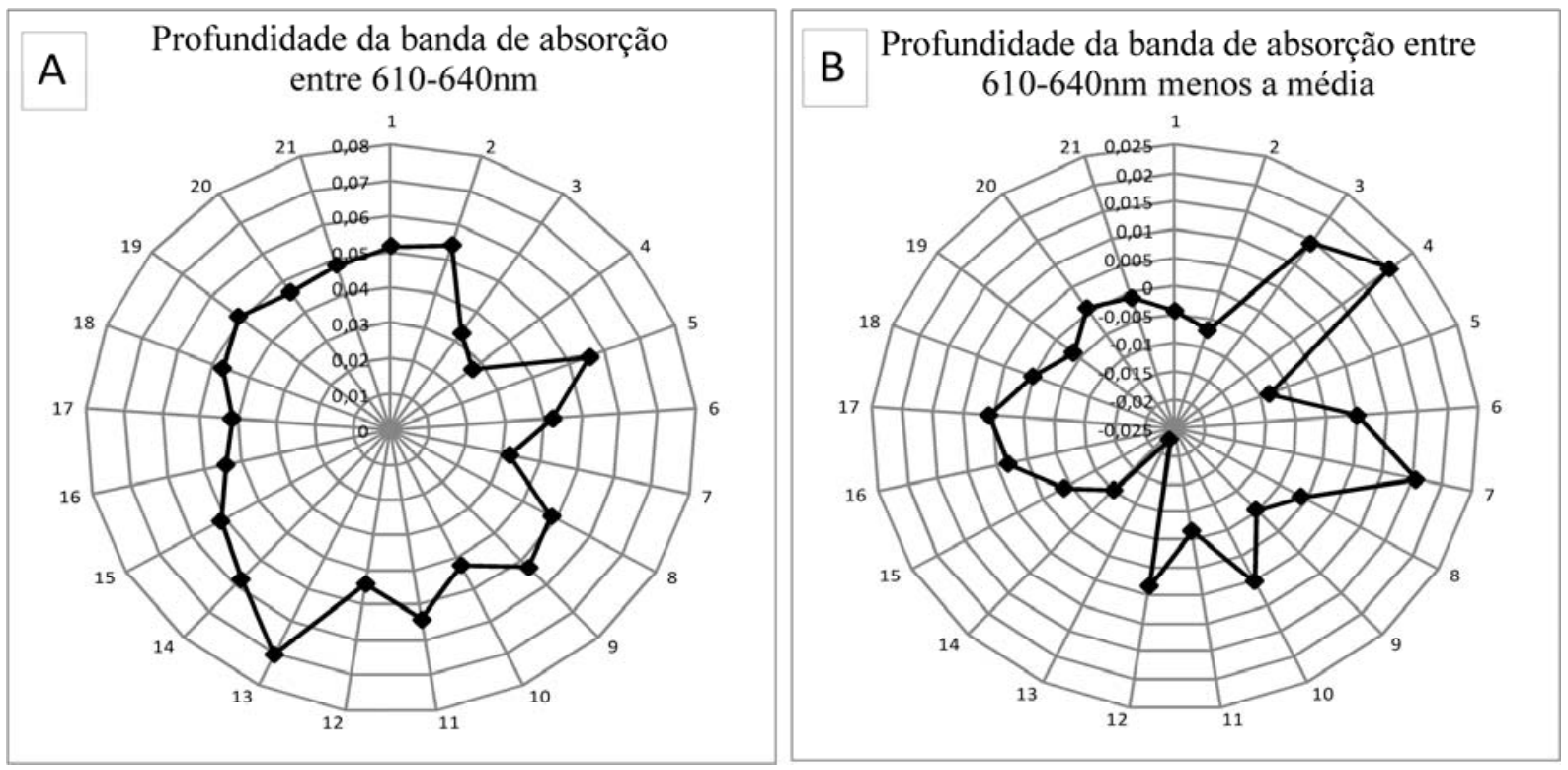

Figura 5. A) Variação da profundidade da banda de absorção centrada em $630 \mathrm{~nm}$ entre o intervalo espectral de 610-640 nm. B) Desvio em relação à profundidade média da banda de absorção centrada em $630 \mathrm{~nm}$ no intervalo espectral de $610-640 \mathrm{~nm}$.

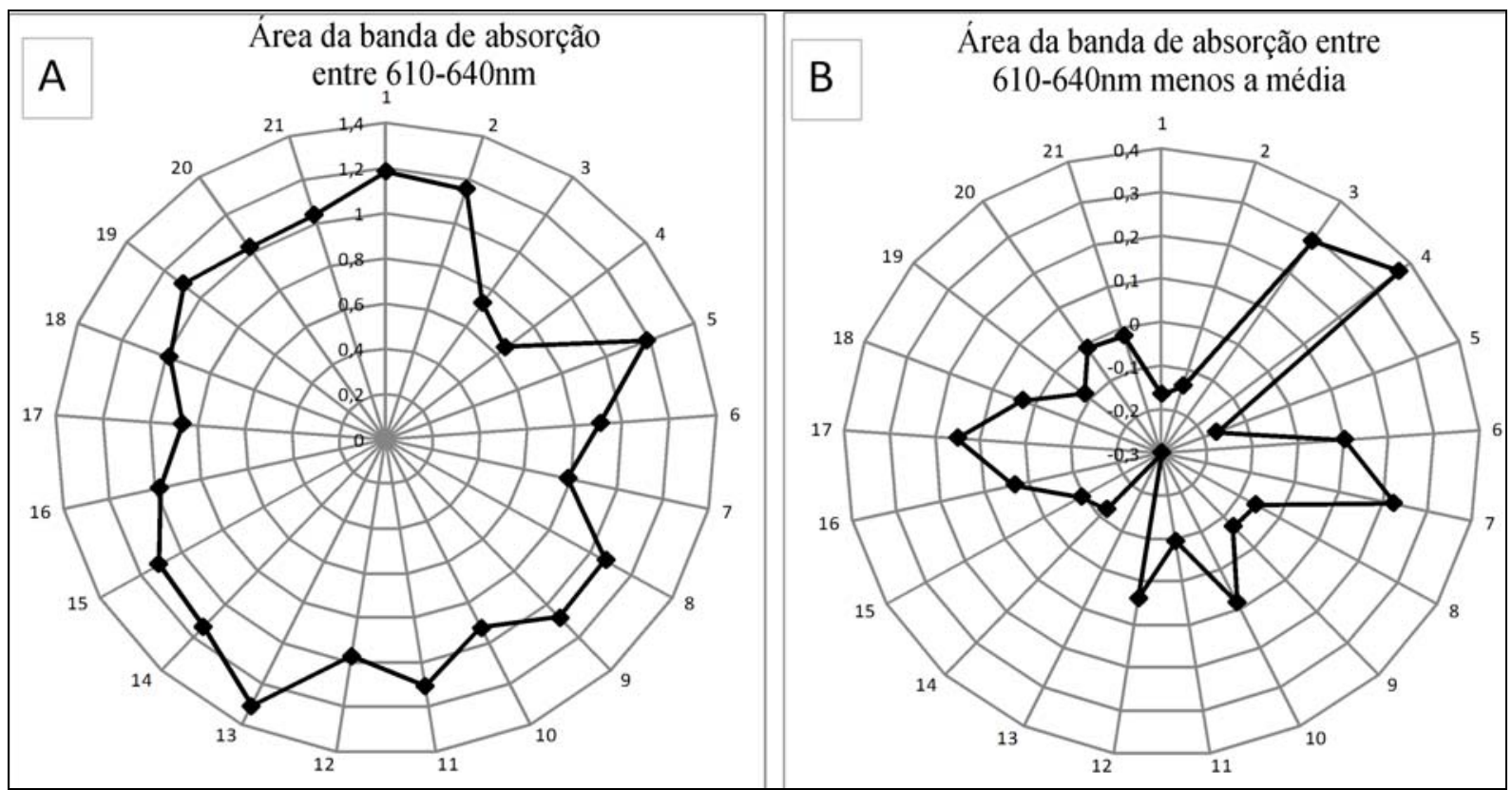

Figura 6. A) Variação da área da banda de absorção entre 610-640 nm. B) Desvio em relação à área média da banda de absorção entre 610-640 nm.

O cálculo do coeficiente de correlação entre a profundidade das bandas de absorção com a profundidade do SD e o TSS apresentou resultados não significativos. Isso também foi 
BREUNIG, F. M.; WACHHOLZ F.; PEREIRA FILHO, W.; RUDORFF, C. M. Análise das propriedades ópticas da água do reservatório Rodolfo Costa e Silva - Itaara, RS, Brasil, usando dados espectrais de campo e imagens orbitais multiespectrais. Ambi-Agua, Taubaté, v. 2, n. 2, p. 88-102, 2007. (doi:10.4136/ambi-agua.28)

encontrado ao correlacionar o TSS e a SD com o parâmetro área da banda de absorção e largura da banda de absorção (Conforme Tabela 3).

Tabela 2. Concentração de TSS e variação da profundidade da SD no reservatório Rodolfo Costa e Silva - RS, no dia 16 de maio de 2006.

\begin{tabular}{cccccc}
\hline Ponto & SD $(\mathbf{c m})$ & TSS $(\mathbf{m g} / \mathbf{L})$ & Ponto & SD (cm) & TSS $(\mathbf{m g l L})$ \\
\hline 1 & 250 & 1,04 & 12 & 255 & 1,71 \\
2 & 252 & 1,34 & 13 & 252 & 2,24 \\
3 & 246 & 0,87 & 14 & 255 & 1,47 \\
4 & 250 & 1,64 & 15 & 250 & 2,14 \\
5 & 255 & 1,44 & 16 & 254 & 1,37 \\
6 & 258 & 1,84 & 17 & 270 & 1,47 \\
7 & 245 & 1,87 & 18 & 281 & 1,41 \\
8 & 261 & 1,07 & 19 & 210 & 2,34 \\
9 & 260 & 1,07 & 20 & 264 & 1,57 \\
10 & 258 & 1,11 & 21 & 240 & 0,97 \\
11 & 248 & 2,27 & & & \\
\hline
\end{tabular}

A baixa correlação obtida pode ser atribuída à baixa variância dos dados e à incerteza das medidas radiométricas e dos parâmetros opticamente ativos presentes na água. Os melhores coeficientes $(0,24)$ foram encontrados ao correlacionar a profundidade da banda de absorção entre 610-640 nm e TSS e entre a profundidade da banda de absorção entre as bandas 400$550 \mathrm{~nm}$ e 400-550 nm com a transparência da água (SD) e, por fim, o valor de 0,34 foi encontrado ao correlacionar a largura da banda de absorção com a transparência da água.

A variabilidade espacial e amplitude da concentração de clorofila no reservatório Rodolfo Costa e Silva podem ser observadas na Figura 7. Ocorre uma baixa amplitude e verifica-se a existência de dois grandes compartimentos, para as clorofilas a, b e c. Um compartimento aquático com menor concentração de clorofila a jusante e com maior concentração a montante do reservatório.

Tabela 3. Valores obtidos da correlação entre parâmetros da aplicação da remoção do contínuo e parâmetros limnológicos.

\begin{tabular}{l|c|c|c|c|c|c}
\hline $\begin{array}{c}\text { Bandas } \\
\text { espectrais (nm) }\end{array}$ & $\begin{array}{c}\text { Cor. } \\
\text { PBA x } \\
\text { SD }\end{array}$ & $\begin{array}{c}\text { Cor. } \\
\text { PBA x } \\
\text { TSS }\end{array}$ & $\begin{array}{c}\text { Cor. } \\
\text { ABA x } \\
\text { SD }\end{array}$ & $\begin{array}{c}\text { Cor. } \\
\text { ABA x } \\
\text { TSS }\end{array}$ & $\begin{array}{c}\text { Cor. } \\
\text { LBA x } \\
\text { SD }\end{array}$ & $\begin{array}{c}\text { Cor. } \\
\text { LBA x } \\
\text { TSS }\end{array}$ \\
\hline $580-700$ & $-0,23612$ & 0,07349 & $-0,04115$ & $-0,07319$ & 0,34326 & $-0,03305$ \\
$400-550$ & $-0,24079$ & $-0,08838$ & 0,20400 & 0,21646 & 0,18851 & $-0,04720$ \\
$610-640$ & $-0,02004$ & 0,24553 & $-0,07751$ & 0,20242 & 0,04539 & 0,01944 \\
$650-680$ & $-0,03669$ & 0,019251 & $-0,02083$ & 0,13420 & 0,04539 & 0,01944 \\
\hline
\end{tabular}

ABA = Área da banda de absorção; PBA = Profundidade da banda de absorção. 


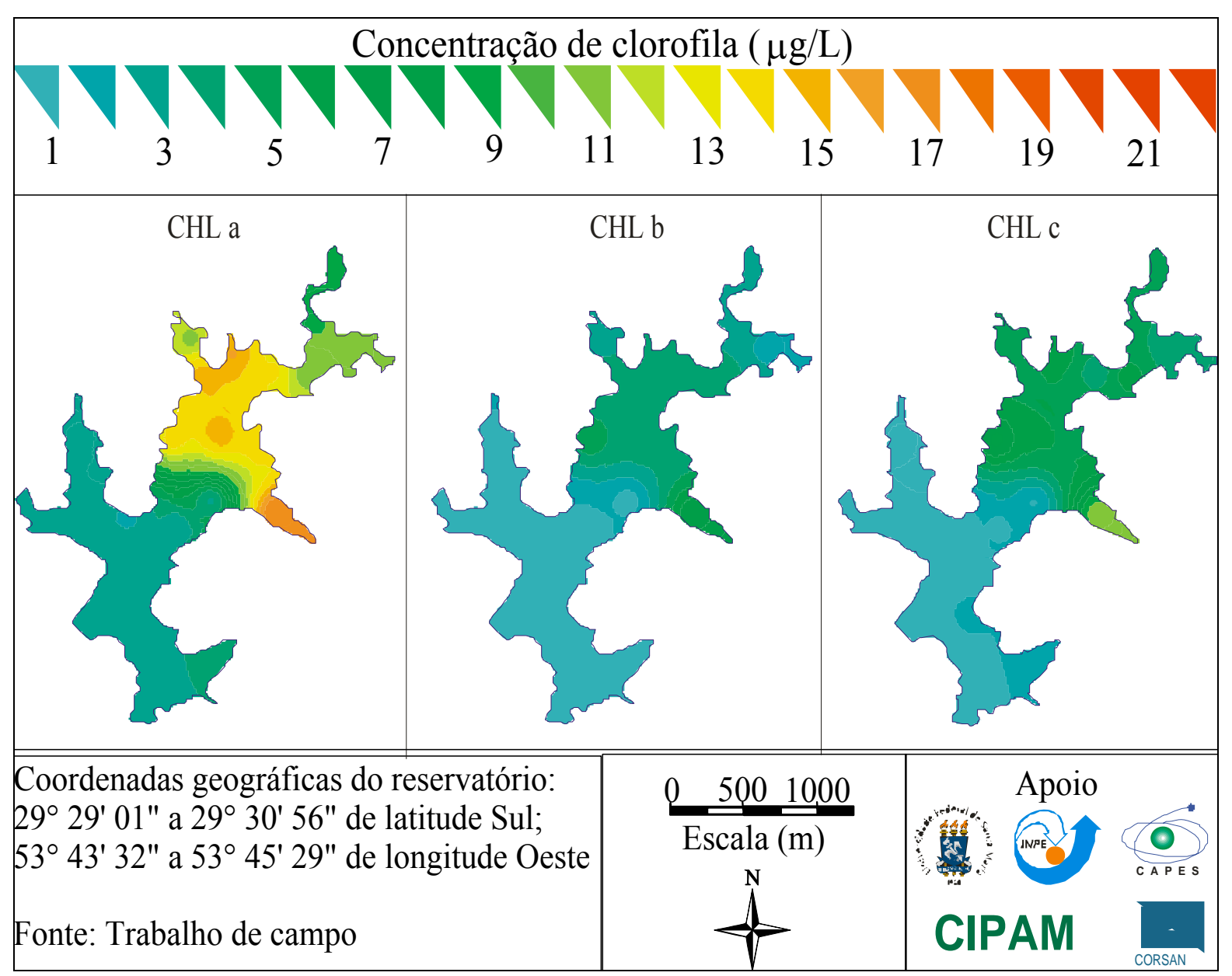

Figura 7. Distribuição espacial da concentração de clorofila a, b e c no reservatório.

A Figura 8A mostra a classificação da imagem TM, e a Figura 8B, a classificação da imagem CBERS. Em ambas as classificações, os resultados não apresentaram boas correlações com os dados de campo. Isso pode ser atribuído à baixa variação das propriedades opticamente ativas da água e aos erros nos processos de correção das imagens. A aplicação de outras técnicas de análise de imagens multiespectrais pode ser vista em Wachholz et al. (2007), que explora os resultados de uma série temporal maior. Entretanto seus resultados concordam com os obtidos neste trabalho.

Por fim, considerando a dificuldade de discriminação de classes de água em reservatórios que apresentam baixa variabilidade da concentração de componentes opticamente ativos, é apresentada a Figura 9 com os valores e amplitude do TSS e transparência da água. Ao analisar a variabilidade dos pontos e sua respectiva concentração de TSS e transparência da água, verifica-se que não são definidos compartimentos aquáticos. 


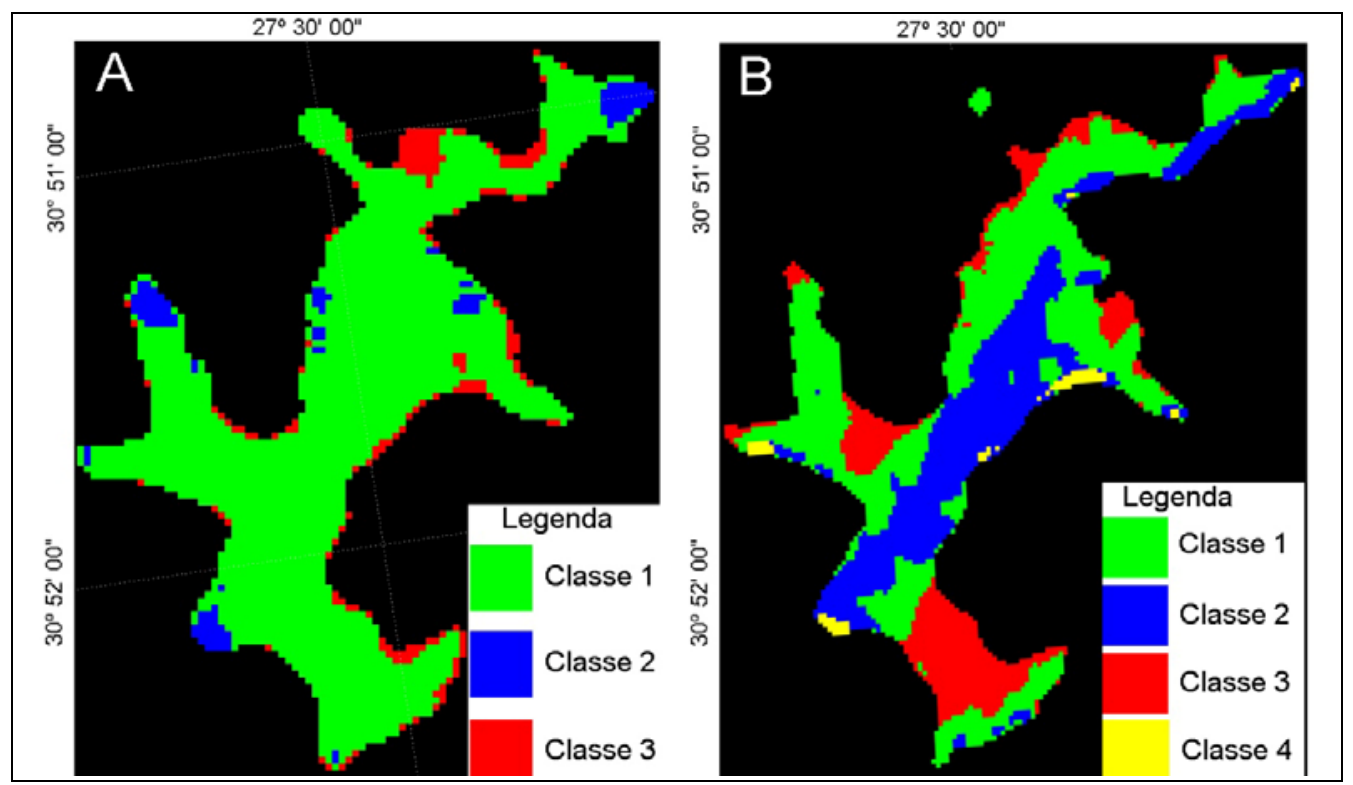

Figura 8. A) Classificação da imagem Landsat TM 5, do dia 5 de maio de 2006. B) Classificação da imagem CBERS-2 CCD do dia 20 de abril de 2006.

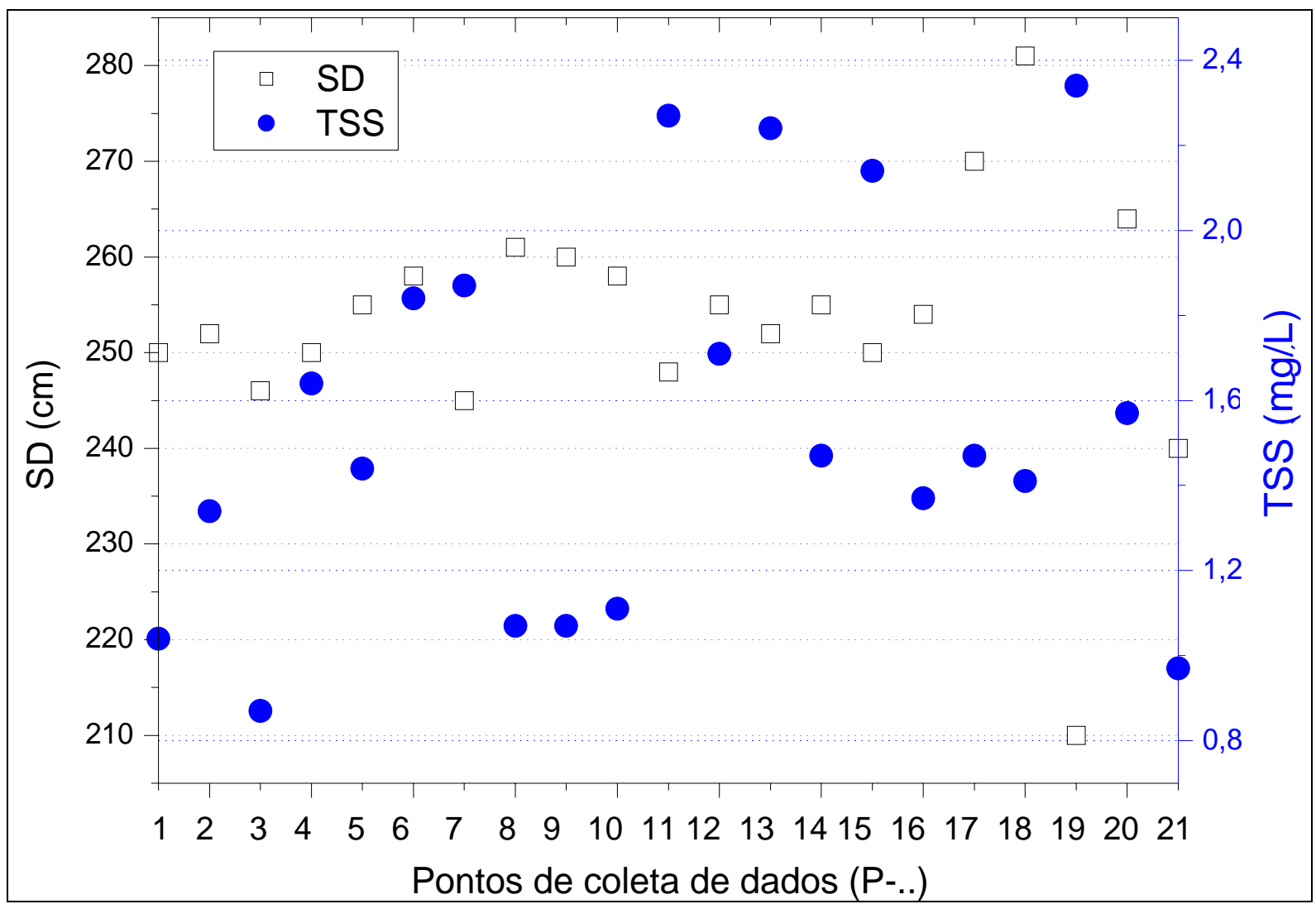

Figura 9. Variação da profundidade do SD e o TSS nos 21 pontos amostrais.

\section{CONCLUSÕES}

A técnica de análise por remoção do contínuo aplicada sobre os espectros de reflectância coletados em campo ajudou a realçar as variações ou feições produzidas pelos componentes opticamente ativos, entretanto não permitiu definir compartimentos aquáticos. Verificou-se 
que existem alguns pontos que se destacaram, entretanto não permitiram definir compartimentos.

Os testes de classificação não supervisionada de imagens multiespectrais orbitais apresentaram resultados pouco aplicáveis no estudo de corpos hídricos de baixa variabilidade dos componentes opticamente ativos (TSS e clorofila), que é o caso do reservatório Rodolfo Costa e Silva, por motivo de limitações de resolução espectral e radiométrica.

No entanto, é necessário continuar testando técnicas para extrair informações mais específicas da reflectância da água por meio de dados hiperespectrais e da qualificação das medidas e correções radiométricas aplicadas aos dados de campo e multiespectrais.

\section{AGRADECIMENTOS}

Os autores agradecem à CAPES pelo apoio financeiro oferecido por meio de bolsa de mestrado e custeio Procad; à Petrobras Ambiental e à CORSAN pelo apoio para a execução deste trabalho.

\section{REFERÊNCIAS}

CLARK, R. N.; ROUSH, T. L. Reflectance spectroscopy: quantitative analysis techniques for remote sensing applications. Journal of Geophysical Research, St. Louis, v. 89, n. B7, p. 6329-6340, 1984.

DEKKER, A. G. Detection of optical water quality parameters for eutrophic waters by high resolution remote sensing. 222f. 1993. Doctor Thesis (Hyperspectral remote sensing of water quality) - Vrije Universiteit, Amsterdam, 1993.

DEKKER, A.G.; MALTUHUS , T. J.; SEYHAN, E. Quantitative modeling of inland water quality for high-resolution MSS system. IEEE - Transactions on geoscience and remote sensing, Piscataway, v. 29, n. 1, p. 89-95, 1991.

ENVI - The Environment for Visualizing Images. Version 4.1 Aug 20, 2004. Boulder: Research Systems, Inc., 2004.

ESTEVES, F. A. Fundamentos de Limnologia. 2. ed. Rio de Janeiro: Interciência, 1998. $602 \mathrm{p}$.

GALVÃO, L. S. Métodos para análise de espectros de reflectância. In: Sensoriamento Remoto: reflectância dos alvos naturais. Brasília: Editora da UNB, 2001. p.225-247. 2001.

GALVÃO, L. S.; PEREIRA, W.; ABDON, M. M.; NOVO, E.; SILVA, J. S. V.; PONZONI, F.J. Spectral reflectance characterization of shallow lakes from the Brazilian Pantanal wetlands with field and airborne hyperspectral data. International Journal of Remote Sensing, London, v. 24, n. 21, p. 4093-4112, 2003.

GARCIA, G. J. Sensoriamento Remoto: princípios e interpretação de imagens. São Paulo: Nobel, 1982.

HONG, T.; TAO, F.; PENGFEI, S. Nonlinear Spectral Similarity measure. In: INTERNATIONAL GEOSCIENCE AND REMOTE SENSING SYMPOSIUM, 2004, Anchorade. Proceedings... [S.1.]: IGARSS, 2004. p. 3272-3275. vol. 5 
JOBIN, W. Sustainable management for dams and waters. Boston: Lewis Publishers, 1998. $288 \mathrm{p}$.

KIRK, J. T. O. Light \& photosynthesis in aquatic ecosystems. 2. ed. Cambridge: University Press, 1996. 509p.

KOKALY, R. F.; CLARK, R. N. Spectrocopic determination of leaf biochemistry using banddepth analysis af absortion features and stepwise multiple linear regression. Remote Sensing of Environment, New York, v. 67, p. 267-287, 1999.

MELACK, J. M; GASTIL, M. Comparison of spectral feature algorithms for remote sensing of chlorophyll in eutrophic lakes. GEOSCIENCE AND REMOTE SENSING SYMPOSIUM, 8-12 ago 1994. Proceedings... [S.1.]: IGARSS, 1994. p. 2363 - 2365. vol.4, 1994.

MUTANGA, O.; SKIDMORE, A. K. Continuum-removed absortion features estimate tropical savanna grass quality in situ. In: EARSEL WORKSHOP ON IMAGING SPECTROSCOPY, 3., 13-16 may 2003, Herrsching. Proceedings... Paris: EARSEL, 2003.

NATIONAL RESEARCH COUNCIL. Committee on Sustainable Agriculture and the Environment in the Humid Tropics. Sustainable Agriculture and the Environment in the Humid Tropic. Washington: National Academic Press, 1993.

Committee on USGS Water Resources Research. Estimating water use in the United States: a new paradigm for the national water-use information program. Washington: National Academic Press, 2002.

NOOMEM, M. F.; MEER, F. D.; SKIDMORE, A. K. Hyperspectral remote sensing for detecting the effects of three hydrocarbon gases on maize reflectance. In: INTERNATIONAL SYMPOSIUM OF REMOTE SENSING OF ENVIRONMENT, 31., 20-24 june 2005, Saint Petersburg. Proceedings... Saint Petersburg: ISRSE, 2005.

NORLING, P.; WOOD-BLACK, F.; MASCIANGIOLI, T.M. Water and sustainable development: opportunities for the chemical sciences: a workshop report to the chemical sciences roundtable. Washington: National Academic Press, 2004.

NOVO, E. M. L. M. Comportamento espectral da água. In: MENESES, P. R.; MADEIRA NETTO, J. S. (Org). Sensoriamento remoto: reflectância de alvos naturais. Brasília: Universidade de Brasília, 2001.

NOVO, E. M. L. M.; BARBOSA, C. C. F.; FREITAS, R. M.; SHIMABUKURO, Y. E.; MELACK, J. M.; PEREIRA FILHO, W. Seasonal changes in chlorophyll distributions in Amazon floodplain lakes derived from MODIS images. Limnology, [S.1.], v. 7, p. 19, 2006.

NUSH, E. Comparison of differents methods for chlorophilla and phaeropigments determination. Arch. Hydrobiol., Stuttgart, v. 14, p. 14-36, 1980.

PASTERKAMP, R.; DEKKER, A. G.; HOOGENBOOM, H. J.; RIJKEBOER, M.; HAKVOORT, J. H. M The effect of the specific inherent optical properties on assessing water quality in Dutch inland waters using matrix inversion. GEOSCIENCE AND 
REMOTE SENSING SYMPOSIUM, 28 june - 2 july, 1999, Hamburgo. Proceedings... Hamburgo: IEEE, 1999. p. 2095 - 2097. vol. 4.

PEREIRA FILHO, W. Integração de dados de campo e sensoriamento remoto no estudo da influência das características da bacia de captação na concentração de sólidos em suspensão em reservatório: o exemplo de Tucuruí. 175 f. 1991. Dissertação (Mestrado em Sensoriamento Remoto) - Instituto Nacional de Pesquisas Espaciais, São José dos Campos, 1991.

PEREIRA FILHO, W; BARBOSA, C. C. F.; NOVO, E. M. L. M. Influência das condições do tempo em espectros de reflectância da água. In: SIMPÓSIO BRASILEIRO DE SENSORIAMENTO REMOTO, 12., 2005, Goiânia. Anais... São José dos Campos: INPE, 2005. p. 415-422.

PU, R.; GE, S.; KELLY, N. M.; GONG, P. Spectral absortion features as indicators of water status in coast live oak (Quercus agrifolia) leaves. International Journal of Remote Sensing, London, v. 24, n. 9, p. 1799-1810, 2003.

RUDORFF, C.M.; NOVO, E.M.L.M.; GALVÃO, L.S. Spectral mixture analysis of inland tropical Amazon Floodplain waters using EO-1 Hyperion. In: IEEE GEOSCIENCE AND REMOTE SENSING SYMPOSIUM, 27., 2006, Denver. Proceedings... Denver: IEEE International, 2006.

SÃO PAULO (Estado). Secretaria de Estado do Meio Ambiente. Companhia de Tecnologia de Saneamento Ambiental. Guia de coleta e preservação de amostras de água. Coord. de Edmundo Garcia Agudo et al. São Paulo: CETESB, 1987.

VAN DER MEER, F. Spectral curve shape matching with a continuum removal CCSM algorithm. International Journal of Remote Sensing, London, v. 21, n. 16, p. 3179$3185,2000$.

VERMOTE, E. F.; TANRE, D.; DEUZE, J. L.; HERMAN, M.; MORCRETTE, J. J. Second simulation of the satellite signal in the solar spectrum (6S): an overview. IEEE Trans. Geosc. and Remote Sens., Piscataway, v. 35, n. 3, p. 675-686.

WACHHOLZ, F.; BREUNIG, F. M.; FONSECA, L. M. G.; SARTOR, S. C. B.; PEREIRA FILHO, W. Identificação de compartimentos aquáticos com imagens CBERS-2 CCD, Landsat TM5 e dados de campo no reservatório Rodolfo Costa e Silva-RS. In: SIMPÓSIO BRASILEIRO DE SENSORIAMENTO REMOTO, 13., 2007, Florianópolis. Anais... São José dos Campos: INPE, 2007. p. 3607-3614. 